\title{
Trabajo y desempleo de los jóvenes del Área Metropolitana del sur de Madrid. Su opinión sobre el mercado laboral ${ }^{1}$
}

\author{
M. a José Aguilera Arilla, M. aㅡ Victoria Azcárate LuXán y \\ M. PILAR GONZÁLEZ YANCI
}

\section{RESUMEN}

La situación actual de la juventud respecto a su inserción en el mundo laboral es compleja y heterogénea. El estudio de las caracteristicas de un amplio grupo de jóvenes en un espacio socioeconómico homogéneo del Área Metropolitana de Madrid, a través de una encuesta, nos permite conocer la realidad de una parte de la juventud y la incidencia que factores como la formación personal y el origen social tienen en la aparición de desequilibrios en el mercado laboral, así como aspectos muy interesantes de la opinión de los jóvenes respecto a aquél y a sus posibles soluciones.

\section{INTRODUCCIÓN}

En los últimos años se aprecian las grandes dificultades que, sobre todo los más jóvenes, tienen para comenzar su vida laboral. La cada vez menor oferta de empleo, entre otras razones por desaparición de industrias tradicionales, y la mayor exigencia de cualificación en la oferta existente, explican parte de aquellas dificultades. Mientras que, por otro lado,

Este articulo se ha realizado a partir del informe correspondiente al proyecto: "Desequilibrios del mercado de trabajo juvenil en el Área Metropolitana Madrileña", elaborado también por los doctores Borderías, Murillo y Santos. 
proliferan como oferta nueva multitud de pequeños empleos, caracterizados por su gran precariedad.

En este artículo se hace un análisis a fondo de un amplio grupo de jóvenes de una zona que, a priori, puede considerarse entre las de mayor problemática, por estar integrada en el Área Metropolitana de una gran ciudad con alta proporción de jóvenes entre sus habitantes, y donde el paro castiga con mayor intensidad.

El objetivo esencial que nos proponemos es analizar el grado de incidencia que pueda tener en la integración de los jóvenes en el mercado laboral y en la aparición de los posibles desequilibrios, tanto su formación como su origen social, medido de forma indirecta. La información que nos brinda la encuesta nos va a permitir, además, un rico conocimiento de la opinión que los jóvenes tienen sobre cuestiones relativas al mundo laboral que nos ayuda a conocer mejor a la juventud.

\section{LA UTILIZACIÓN DE LA ENCUESTA COMO FUENTE DE INFORMACIÓN}

El mercado laboral juvenil, al ser una parte integrante del mercado laboral nacional, participa de sus características fundamentales (crisis del pleno empleo, escasez de la oferta de trabajo, terciarización de la economía, lo que conlleva una polarización en ese sector de las ofertas de trabajo, etc.) pero, además, posee otras que le confieren una marcada personalidad.

En primer lugar, los integrantes de ese mercado laboral son personas entre 15 y 29 años, es decir jóvenes, lo que le confiere una serie de características positivas y negativas derivados de la propia fase vital de aquéllos. Los aspectos negativos que presenta dicho mercado se resumen en la escasa oferta de empleo, característica común a todo el mercado laboral, pero que se acentúa más en el caso del empleo juvenil, sobre todo por la falta de experiencia, muchas veces solicitada, y derivada igualmente de la propia inexperiencia vital de sus componentes. Los aspectos positivos son, sin embargo, más numerosos y son también consecuencia de su juventud, ella les proporciona mejor salud, mayor vitalidad, fuerza y energía, menores lazos de dependencia familiares, menores condicionamientos para desplazarse espacialmente, etc. A pesar de ello, los jóvenes, en general, muestran una cierta inseguridad y confusión, así 
como manifiestan un escaso conocimiento de su propia problemática laboral, corroborada por la ambigüedad de sus actitudes y comportamientos, no sólo ante el mercado laboral, sino también incluso ante otras circunstancias de la vida (emancipación, matrimonio, hijos, etc.).

El análisis de las características del mercado laboral juvenil, en general, puede hacerse a través de algunas estadísticas oficiales, pero, es mucho más difícil extraer de ellas relaciones entre variables pertenecientes a un mismo individuo, haciendo un seguimiento de su propia vida y de la de sus familiares, para poder deducir la existencia o no de relaciones entre situaciones pasadas y futuras de un mismo sujeto. Precisamente, para estudiar estas relaciones la mejor fuente de información es la encuesta y por eso se decidió su utilización para lograr alcanzar los objetivos propuestos.

La idea fundamental de partida venía a ser que las características de cada sujeto debian estar relacionadas con él mismo: su edad, su nivel de estudios, su situación laboral, etc., pues había que ponerlas en relación con las de sus padres, él nos debía de decir su propia situación laboral, sus actitudes ante los diferentes problemas del mercado laboral, su opinión respecto a algunos aspectos del mismo, etc. Es decir, las variables que necesitábamos para el estudio eran personalizadas, aunque por supuesto anónimas, y habían de poderse relacionar con las de sus padres. Ello nos confirmó, todavía más, el que nos planteásemos la necesidad de hacer un cuestionario "ad hoc".

El planteamiento del cuestionario se realizó en función de los objetivos que se perseguian. Preguntas como ¿reproducen los hijos la situación educativa y laboral de los padres? ¿la segunda generación mejora en alguno de estos dos aspectos? ¿qué incidencia tiene una y otra en la actual situación de los hijos? Por un lado, se trataba de establecer relaciones entre el nivel de formación de los jóvenes y el alcanzado por sus padres, con la situación laboral actual de los primeros. Por otro, se tenía que relacionar la situación laboral de los padres y el nivel de formación de los jóvenes, igualmente con la actual situación laboral del hijo. Ambas relaciones podían plantearse como hipótesis de trabajo que habia que constatar. A priori, parecía que podía existir una cierta correlación, puesto que en el tratamiento analítico de algunas de las variables de las estadísticas oficiales, aparecen más o menos significativamente relacionadas. También, algunos estudios anteriores y la observación directa de varios casos concretos, mostraban una clara incidencia del origen social familiar en la formación y postura ante el trabajo de los jóvenes. Además, se pretendía co- 
nocer la existencia de otras posibles relaciones entre las características sociodemográficas de los jóvenes, su nivel de formación y su situación laboral. También se pretendía descubrir cuál era su conocimiento del mercado de trabajo juvenil y de las posibles ayudas que podian recibir para su integración en el mismo.

El cuestionario se componía de cuatro grandes apartados:

1. Características sociodemográficas de los jóvenes.

2. Nivel de formación alcanzado por los jóvenes y sus padres.

3. Integración de los jóvenes en el mercado de trabajo.

4. Valoración del mercado de trabajo juvenil.

Algunos de estos grandes capítulos se plantearon a todos los jóvenes encuestados. Otros comprendian preguntas que se hacían sólo a los que se hallaban en una situación concreta. Por ejemplo, en el capítulo III se subdividieron las preguntas entre los que estaban trabajando (ocupados) y los que no lo hacian (inactivos y parados). También su situación anterior, si había cambiado en el tiempo, fue motivo de interrogación concreta. Asimismo, el hecho de trabajar por cuenta ajena o por cuenta propia establece algunas diferencias por lo que su análisis debía hacerse por separado. La satisfacción personal que sentía el encuestado con el trabajo que desempeñaba en aquellos momentos, fue otra de las preguntas más fundamentales. La valoración del mercado laboral juvenil se midió con preguntas relacionadas con la información que poseían del mismo, tanto relacionadas con los distintos tipos de trabajo existentes en esos momentos, como con las instituciones, programas y oficinas que podían brindarles distintas ayudas a su formación y empleo. Por último, se quiso obtener información sobre las ideas que pudieran poseer los jóvenes, a fin de tratar de solucionar los problemas que se plantean a la juventud en su incorporación al mercado de trabajo, para lo cual se dejó abierta la posibilidad de respuesta.

Todo esto se resumió en un total de 66 preguntas cuya respuesta podía llevarse a cabo, presumiblemente, en un tiempo máximo de treinta minutos. El tamaño de la muestra utilizado fue de 600 jóvenes, que tras la depuración de los cuestionarios no válidos, quedaron en un total de 542 a la hora de tratar estadísticamente la información. Los jóvenes a los que se debía interrogar tenian que reunir, además de la edad (15-29 años), el requisito de no encontrarse cursando estudios reglados. 
La encuesta se realizó en los municipios de Getafe y Fuenlabrada, pertenecientes al Área Metropolitana Sur I, socioeconómicamente homogénea conforme a un análisis previo de diferenciación del Área Metropolitana de Madrid (AGUILERA et al., 1996).

\section{CARACTERISTICAS SOCIODEMOGRÁFICAS}

El colectivo de jóvenes encuestados presenta una pequeña mayoría de varones, son más numerosos en los primeros años, fundamentalmente entre los 18 y 20 años, aunque las mujeres son más entre los 27 y 29 años (figura 1). La inmensa mayoría son solteros, y conviven con su familia en un núcleo de tamaño medio; sólo un $11 \%$ dice convivir con su pareja, casados o no. En su mayoría también, son dependientes $(66 \%)$ esencialmente de sus padres. La falta de trabajo y de recursos económicos para pagar una vivienda, son las causas más citadas como responsables de esta situación.

El origen social de estos jóvenes, deducido de la profesión y nivel de estudios de padre y madre, resulta homogéneo y en conjunto no muy ele-

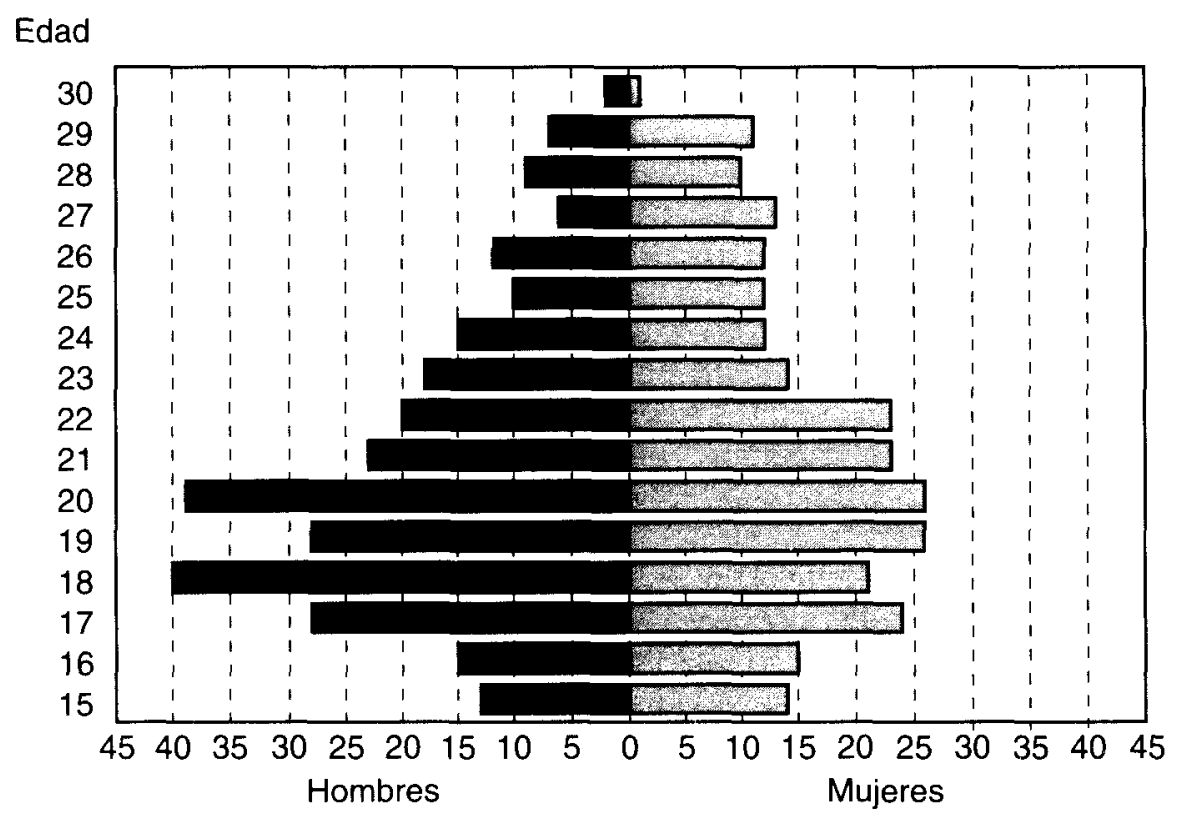

Figura 1. Población joven. Encuesta Área Metropolitana Sur 1995. 
vado. La mayoría de los padres trabajan en puestos que requieren escasa cualificación y las madres se declaran amas de casa; si la mujer trabaja, suele hacerlo como empleada de hogar, la mayoría de las veces como asistentas a tiempo parcial. El nivel educativo de los padres no supera generalmente el de graduado escolar o primera etapa de E.G.B. y tan sólo un $2 \%$ dice poseer titulación universitaria. El nivel de estudios materno es aún, si cabe, más bajo y sólo tres de las madres dicen poseer estudios superiores.

La renta familiar mensual se concentra en valores inferiores a las 250.000 pesetas, que la mayoría de las veces es obtenida por más de una persona del conjunto familiar.

Los jóvenes se consideran, sin embargo, mayoritariamente (68\%) como de clase media y sólo un $22 \%$ como de clase media-baja. Sus ingresos personales son inexistentes para casi la mitad de los encuestados, recibiendo de su familia de 500 a 5.000 pesetas semanales para sus gastos. Los demás manifiestan obtener ingresos que oscilan entre las 25.000 y las 150.000 pesetas mensuales en concepto de remuneración por su trabajo.

El nivel de formación adquirido por los jóvenes, que han terminado la primera enseñanza obligatoria, muestra que la mayoría se quedaron en esa primera etapa de estudios. Sólo un $5 \%$ de los encuestados dice estar en posesión de un título universitario, ello nos indica, al menos cuantitativamente hablando, la mejora de su nivel de formación respecto al de sus padres $(2 \%)$.

Sin embargo, y pese al abandono de la enseñanza reglada, muchos de estos jóvenes continúan estudiando para mejorar su formación de cara a la búsqueda de un puesto de trabajo. Algunos manifiestan estar llevando a cabo cursos como: informática, idiomas, mecanografía y secretariado. Otros muestran un interés especial en recibir cursillos prácticos de peluquería, fontanería, cerrajería, en cuanto a profesiones tradicionales, o de seguridad y vigilancia, por la cada vez mayor demanda existente en esos campos laborales.

La edad a la que abandonaron los estudios reglados supone una preciosa información añadida. Más de la mitad del total dejaron sus estudios antes de los 20 años y más de una tercera parte antes de los 16. Si a ello añadimos que la inmensa mayoría manifiesta que fue la falta de motivación por el estudio lo que les indujo a dejarlo, veremos cómo puede subyacer en la decisión de abandono, la ausencia o escasez de formación 
de su núcleo familiar. En este contexto los estudios se consideran, a menudo, como algo que, aunque valorado, no parece imprescindible en la reproducción de los esquemas de partida. El bajo nivel de estudios declarado por padre y madre presenta una gran relación con la edad de abandono de los estudios del hijo, ésta es más temprana cuanto menor es la formación de sus padres. Junto a esto, la necesidad de nuevos ingresos en su núcleo familiar, el desánimo y el fracaso escolar, completan la panorámica de causas que les llevan al abandono o interrupción de su vida de estudiante. Estas causas son a su vez indicadores de la escasa valoración que tiene para estos jóvenes la formación cultural, lo que, sin disculpar la gran influencia del propio sistema educativo (asignaturas, contenidos, profesorado...) vuelve a tener relación con la principal fuente tradicional de transmisión de valores, la familia.

La opinión que presentan los jóvenes respecto a la enseñanza que han recibido es, sin embargo, bastante buena en general, al menos eso es lo que indica una gran mayoría. Otros muestran cierto desencanto y sólo un $5 \%$ la califica de mala. Por otro lado, son algo más de la décima parte los que señalan que no les va a ser de ninguna utilidad a la hora de encontrar trabajo. Es, por todo esto, bastante evidente que la cultura no se valora en sí misma, sino en función de las exigencias para un puesto de trabajo.

\section{INTEGRACIÓN DE LOS JÓVENES EN EL MERCADO LABORAL. INCIDENCIA DEL ORIGEN SOCIAL Y NIVEL DE FORMACIÓN}

En el análisis de la situación de los jóvenes de cara a su integración en el mercado laboral, se definen dos grupos netamente diferenciados: aquéllos que declaran no estar trabajando y que suponen el $59 \%$ del conjunto, frente al $41 \%$ restante que sí tenía una ocupación determinada. Esbozaremos a continuación algunos rasgos característicos de ambos grupos.

Dentro del grupo que no trabaja casi la cuarta parte no manifiesta ningún deseo de buscar trabajo, por lo que se les puede considerar como inactivos -en sentido económico - por no haber trabajado ni buscado empleo. El resto sí ha tenido algún contacto, aunque esporádico, con el mundo laboral.

La experiencia laboral de estos jóvenes es muy variada y su análisis se puede efectuar desde diversos ángulos. Es un fenómeno relativamente 
frecuente que la experiencia de muchos de estos jóvenes se reduzca a trabajos de ayuda familiar debido a la escasez de oportunidades de empleo. Este tipo de trabajo, conocido tradicionalmente como «trabajo de ayuda familiar", es muy común en el sector servicios, y en él se insertan la mayor parte de aquéllos. Esta situación de dependencia a menudo supone un arma de doble filo: si bien, por un lado, tiene la ventaja de porporcionar una cierta experiencia laboral, que fuera del entorno familiar estos jóvenes no encuentran, por otro, hace que su proceso de emancipación se pueda retrasar.

Por el contrario, otros jóvenes sí han tenido una experiencia laboral fuera del entorno familiar y previa a su situación actual de paro. La inmensa mayoría tuvo un empleo sin contrato, de cuya existencia se enteró principalmente a través de amigos y familiares, precisamente por tratarse generalmente de un trabajo en algún modo irregular o sumergido. Los trabajos que realizaron fueron en su mayor parte eventuales, esporádicos 0 temporales, es decir, de corta duración, lo que corrobora la inestabilidad del mercado laboral juvenil.

En el caso de aquellos jóvenes que en la actualidad no están ocupados, pero que anteriormente tuvieron una trabajo en condiciones legales, es decir, con contrato, la duración del mismo vuelve a ser un indicador de la precariedad en el empleo. Por lo general se trata de trabajos que requieren escasa formación y, en su gran mayoria, están encuadrados en el sector servicios, más concretamente, en el comercio.

Como hemos visto anteriormente, existe otro importante grupo de jóvenes que si estaba trabajando en el momento de hacer la encuesta. La inmensa mayoría comenzó a hacerlo después de los 16 años, lógicamente por tratarse de la edad que la legislación actual española marca como necesaria para poder empezar a trabajar. Frente a ellos, un reducido número de jóvenes no empezó a trabajar hasta cumplidos los 23 años, lo que podria indicar una mayor preparación o incluso haber realizado estudios superiores, ya que es justamente a esa edad cuando suelen finalizar los estudios de licenciatura.

Asimismo, existe precariedad en el empleo y una notable movilidad corroboradas por el elevado número de contratos anteriores al actual que han tenido muchos jóvenes. Además, por un lado han trabajado en varias empresas $y$, por otro, han tenido varios contratos en la misma empresa. 
Son muy pocos aquellos jóvenes que trabajan por cuenta propia. En este sentido, resulta muy ilustrativo destacar que tan sólo un $10 \%$ de éstos ha recibido alguna ayuda oficial por ser joven que, como promoción al autoempleo, se ofrece desde diversas instituciones estatales o de la CAM. Por lo general, estos trabajos realizados por cuenta propia suelen tener un carácter ocasional o eventual.

Por el contrario, son mayoría los jóvenes que trabajan por cuenta ajena, al igual que sucede en el mercado laboral en general. Éstos realizan un trabajo de forma continuada y, normalmente, a tiempo completo. En un porcentaje elevado poseen un contrato para realizar su trabajo. Sin embargo, la gran precariedad que existe en el mercado laboral juvenil se manifiesta en la exigua proporción de contratos fijos: son relativamente pocos los que consiguen este tipo de contrato, al menos en los primeros períodos de su actividad laboral.

En esta misma línea de precariedad en el empleo, está la duración de los contratos: apenas superan el $10 \%$ aquéllos de más de cuatro años y, por tanto, que ofrezcan visos de cierta estabilidad.

Estos jóvenes trabajan en empresas de pequeño tamaño, con absoluto predominio de las que se dedican al sector servicios. Debido a la fuerte carencia de prácticas que existe en el actual sistema educativo, la juventud considera muy positivamente la formación recibida a cargo de la empresa en la que trabaja, ya sea con o sin remuneración, como medio para adquirir una experiencia y formación práctica.

No obstante, destaca, por otra parte, el notable grado de satisfacción que estos jóvenes muestran ante el trabajo que realizan, y en caso de no estar satisfechos, suele ser por razones económicas y no por el tipo de ocupación que desempeñan. A pesar de ello, tienen una idea de provisionalidad y manifiestan deseo de cambiar de empleo, aunque sólo la mitad lo busque y se prepare para ello.

Por último, la gran mayoría trabaja en su propio municipio. Esta cercanía de su domicilio al puesto de trabajo no deja de ser un dato satisfactorio en el panorama laboral no muy halagüeño que poseen estos jóvenes del Área Metropolitana Sur madrileña.

En el complejo mundo laboral actual, en el que existe una escasez de la oferta de trabajo unida a una creciente terciarización, especialización y aumento del nivel de exigencia que, como hemos comentado, afecta de manera muy especial a los más jóvenes, es de sumo interés analizar la in- 
cidencia que factores como la situación familiar de partida y la propia formación de las personas, puedan tener en la aparición de desequilibrios en el mercado laboral.

En el grupo de jóvenes encuestados del Área Metropolitana Sur de Madrid, encuadrada entre las de más bajo nivel social y mayor problemática juvenil, al poner en relación las más significativas variables de origen social, formación y situación laboral, encontramos que se dan determinadas correlaciones que avalan la hipótesis inicial de la incidencia negativa de la escasa formación educativa en la actual situación laboral juvenil (cuadro 1).

\section{CUADRO 1. SITUACIÓN LABORAL SEGÚN NIVEL DE ESTUDIOS}

\begin{tabular}{|c|c|c|c|c|c|c|}
\hline \multirow{2}{*}{ NIVEL ESTUDIOS } & \multicolumn{2}{|c|}{ TRABAJA } & \multicolumn{2}{|c|}{ NO TRABAJA } & \multicolumn{2}{|c|}{ TOTAL } \\
\hline & $\mathrm{N} .{ }^{\circ}$ & $\%$ & N. ${ }^{\circ}$ & $\%$ & $\mathrm{~N}^{\circ}$ & $\%$ \\
\hline Ninguno acabado ......... & 2 & 50,0 & 2 & 50,0 & 4 & 100,0 \\
\hline Graduado escolar......... & 13 & 48,0 & 14 & 52,0 & 27 & 100,0 \\
\hline 2. Etapa EGB ............... & 90 & 45,9 & 105 & 53,6 & 196 & 100,0 \\
\hline FP I $\ldots \ldots \ldots \ldots \ldots \ldots \ldots \ldots \ldots \ldots \ldots \ldots \ldots \ldots$ & 12 & 28,6 & 30 & 71,4 & 42 & 100,0 \\
\hline FP $\|$ & 21 & 47,7 & 23 & 52,3 & 44 & 100,0 \\
\hline BUP & 32 & 34,8 & 60 & 65,2 & 92 & 100,0 \\
\hline COU & 27 & 36,5 & 47 & 63,5 & 74 & 100,0 \\
\hline \multicolumn{7}{|l|}{ Primer ciclo Univ. } \\
\hline Grado medio................ & 18 & 54,5 & 15 & 45,5 & 33 & 100,0 \\
\hline \multicolumn{7}{|l|}{ Segundo ciclo Univ. } \\
\hline Doctorado......... & 13 & 48,0 & 14 & 52,0 & 27 & 100,0 \\
\hline TOTAL $\ldots \ldots \ldots \ldots \ldots \ldots$ & 228 & 42,1 & 310 & 57,7 & 539 & 100,0 \\
\hline
\end{tabular}

En líneas generales se observa que, al igual que sucede en la sociedad en la que se integran, trabajan en mayor proporción los hombres que las mujeres, los de más edad que los más jóvenes y los casados que los solteros (cuadro 2).

CUADRO 2. SITUACIÓN LABORAL POR SEXO

\begin{tabular}{|c|c|c|c|c|c|c|}
\hline \multirow{2}{*}{ SEXO } & \multicolumn{2}{|c|}{ TRABAJAN } & \multicolumn{2}{|c|}{ NO TRABAJAN } & \multicolumn{2}{|c|}{ TOTAL } \\
\hline & $\mathrm{N} .^{\circ}$ & $\%$ & $\mathrm{~N} .{ }^{\circ}$ & $\%$ & N. ${ }^{\circ}$ & $\%$ \\
\hline Hombres.. & 136 & 47,7 & 148 & 52,3 & 285 & 100,0 \\
\hline Mujeres .......................... & 92 & 36,0 & 165 & 64,0 & 257 & 100,0 \\
\hline TOTAL ................... & 228 & 42,1 & 313 & 57,7 & 542 & 100,0 \\
\hline
\end{tabular}


Considerando como indicadores del origen social de los jóvenes el nivel educativo y la ocupación de los padres, se aprecia que cuanto mayor es el nivel de estudios del padre y, más aún el de la madre, los hijos trabajan en menor proporción, y sobre todo entran en el mercado laboral más tardíamente que aquéllos que tienen padres con los niveles educativos más bajos (figura 2). Igualmente se observa que trabajan desde edad más temprana los que proceden de familias con menor renta (figura 3). La ocupación de los padres, en cambio, presenta una escasa incidencia en la situación laboral de los hijos.

La formación personal de los jóvenes resulta, sin duda, mucho más significativa en la aparición de los desequilibrios citados. Los que tienen

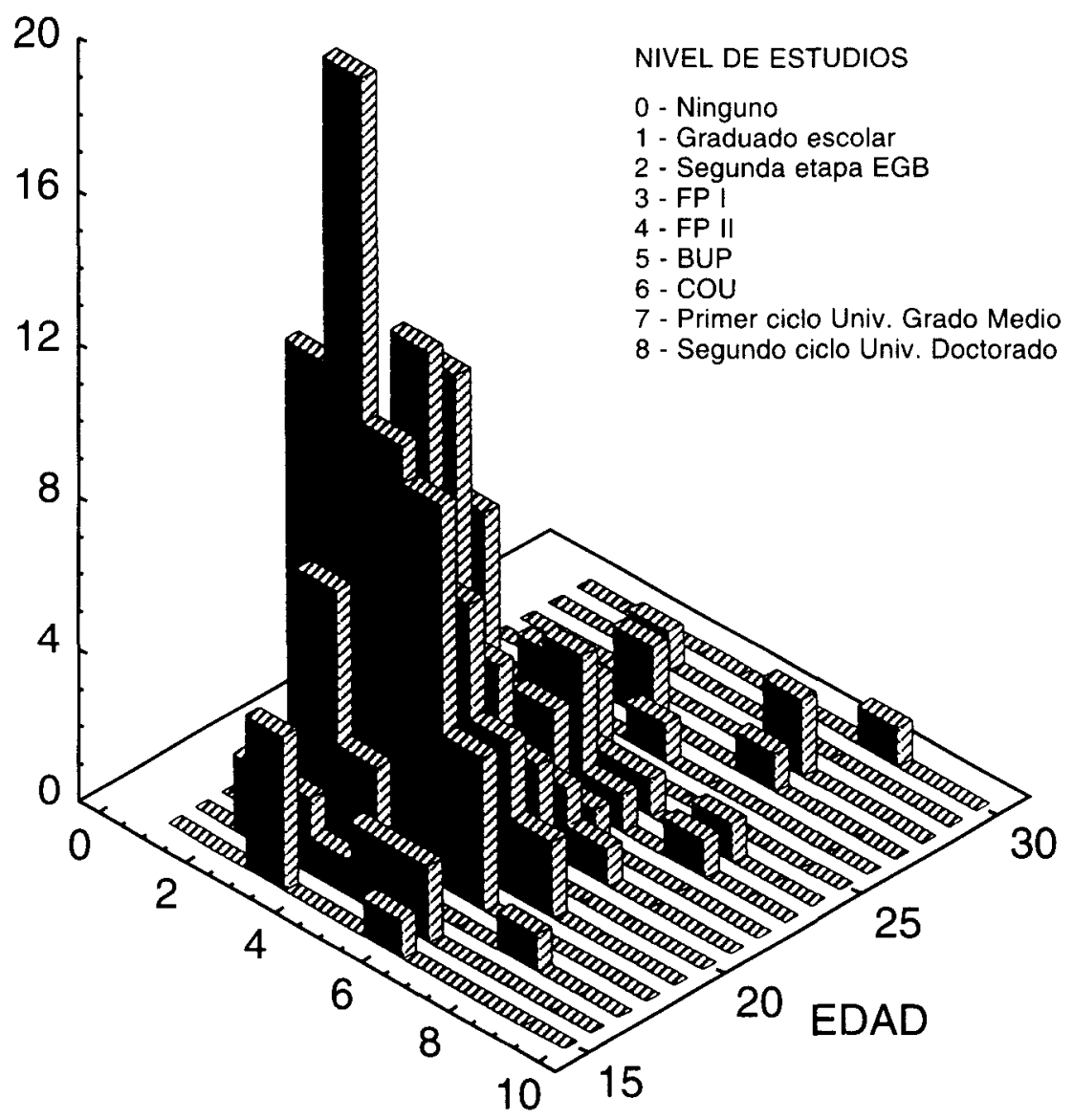

Figura 2. Nivel de estudios del padre y edad de comienzo de trabajo del hijo. 


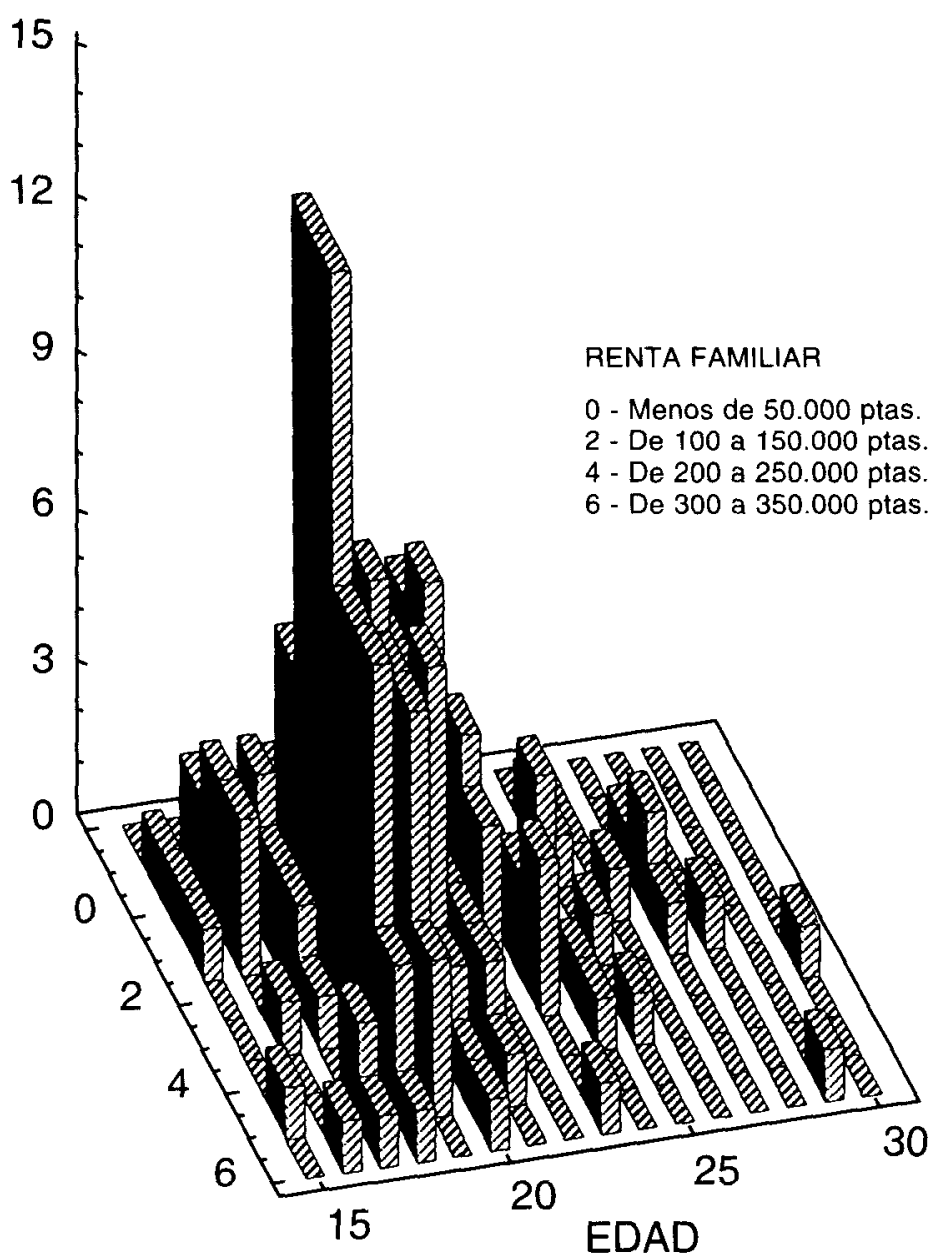

Figura 3. Edad de comienzo de trabajo según renta familiar.

más alto nivel de estudios son los que poseen mayor proporción de contratos fijos, y los que ocupan puestos de más nivel y con sueldos más elevados (figura 4), siendo de ellos los que poseen titulación de grado medio o primer ciclo universitario, los que presentan mayor porcentaje de ocupados. No obstante lo cual hay algunas excepciones, como un pequeño porcentaje de titulados superiores que ocupan puestos de obreros sin cualificar, lo que supone una evidente situación de infrautilización y justifica la frustración que muestran. En definitiva, los que tienen más edad y preparación son los que presentan una situación laboral de mayor estabilidad. 


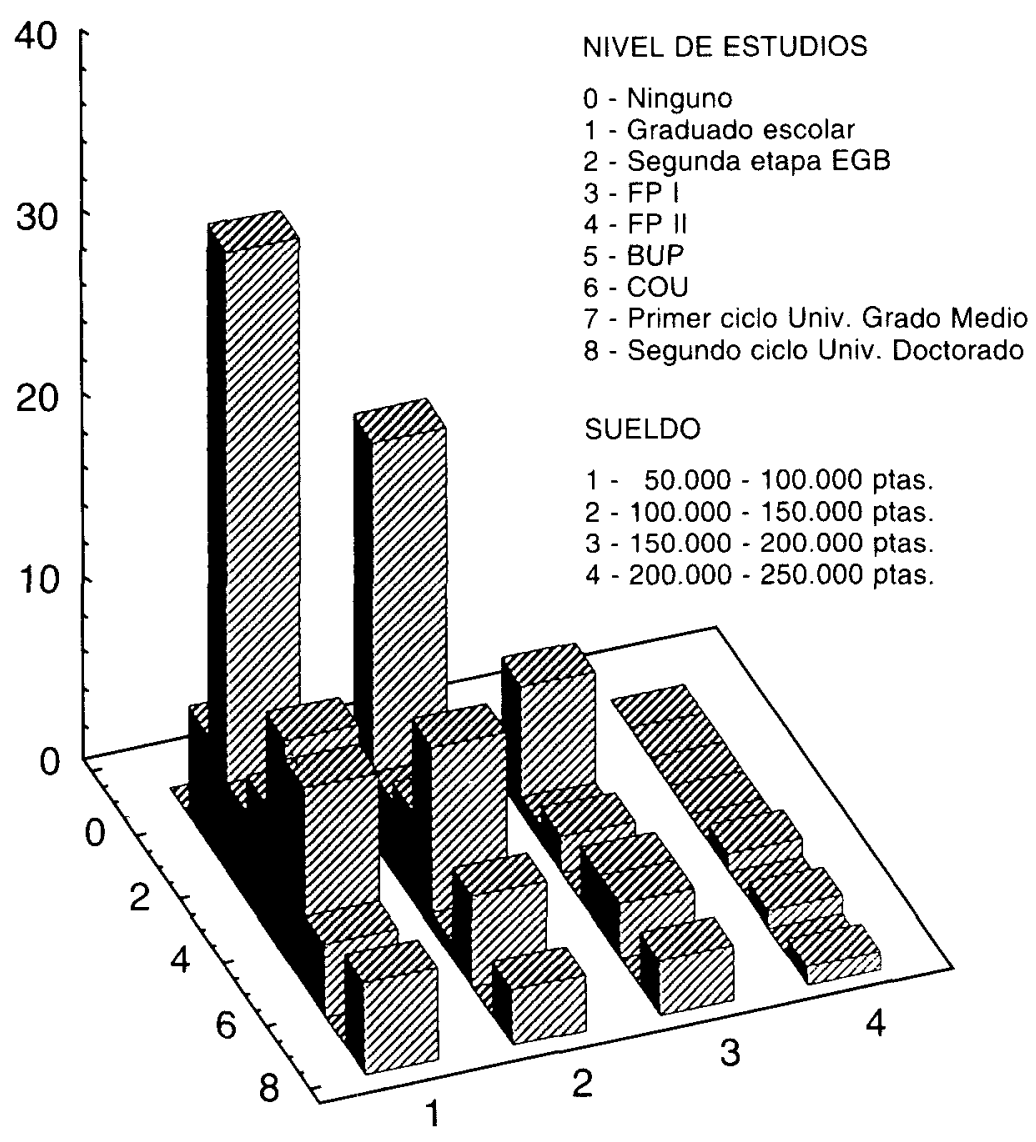

Figura 4. Nivel de estudios y sueldo.

En el extremo opuesto, los de menor titulación ocupan los puestos de inferior categoria y con mayor frecuencia poseen contratos de corta duración y mayor número de ellos. A pesar de lo cual, muestran un sorprendente grado de satisfacción en el trabajo que realizan, lo que indica que hay una valoración del hecho de contar con un empleo y de disponer de un dinero, aunque no sea mucho, mientras continúan viviendo con sus padres.

Por tanto, del análisis de este grupo se deduce la incidencia que tiene la formación en la situación laboral, al manifestarse, entre un variado número de casos, la presencia de dos extremos en los que se muestra la situación más contrastada. Los de mayor titulación y preparación son los que tienen la mejor situación, con mayor estabilidad en el empleo, mejor 
sueldo y ocupan puestos de más nivel, mientras que en el extremo opuesto los menos preparados ocupan los puestos inferiores, de menor remuneración y tienen más precariedad en el empleo.

\section{POSICIÓN DE LOS JÓVENES RESPECTO AL MERCADO LABORAL}

Desde hace unos años en la población española está muy presente la preocupación por el problema del empleo, o si lo preferimos del paro, que son dos caras de la misma moneda. Estamos acostumbrados a que ante la pregunta de cuál es el principal problema del país sea abrumadora la mayoría que responde que el "paro» o algo similar, con una especial atención a que encuentren trabajo los jóvenes. Esta preocupación genera entre estos últimos un sentimiento hacia el futuro y hacia el trabajo de inseguridad, confusión y precariedad muy generalizado.

El marco de referencia social actual presenta algunas caracteristicas como el progresivo envejecimiento de la población, la tendencia a que cada día un menor número de ocupados tenga que sostener a un mayor número de dependientes, el descenso de la demanda de personas con una cualificación incluso universitaria, la «aceptación» de una "economía sumergida" y del "trabajo precario", la incapacidad del sistema para resolver el paro real, la certeza del aumento del paro sectorial (por ejemplo en la enseñanza por descenso demográfico de los menores, o el de determinadas industrias por cierre de fábricas), el probable descenso de la calidad y nivel de vida con respecto al de los padres y un largo etcétera, que provocan en la juventud unas tendencias constatadas a refugiarse en la familia de origen por más tiempo, a sentir escepticismo por el asociacionismo y por la política, a tender a un progresivo individualismo, al alargamiento de su etapa de dependencia y a evitar contraer compromisos sociales como matrimonio o hijos; a sentirse poco útiles a la sociedad y a perder el sentido social del trabajo, que se ve como un instrumento de supervivencia personal, a buscar prolongar la etapa de juventud y de estudiante de forma artificial y hasta a marginarse de la sociedad... (ELZO, 1990 pp. 96-97).

Por todo esto es muy interesante conocer la opinión de un nutrido conjunto de jóvenes, heterogéneo en cuanto que abarca un amplio abanico de edades (de quince a veintinueve años) y de situaciones (que trabajan o están parados, que han abandonado sus estudios a diversas edades, 0 que han concluido estudios superiores, que tienen trabajo fijo, o que de- 
sempeñan o han desempeñado pequenos trabajos eventuales) y que, en cambio, es bastante homogéneo desde un punto de vista social, ya que pertenecen a una misma zona del Área Metropolitana de Madrid, y a un entorno social de clase media, media-baja, según su propia consideración, donde, no obstante, se encuentran diversas situaciones económicas y sociales. A través de un conjunto de preguntas encaminadas a conocer la valoración que hacen del mercado de trabajo, hemos podido constatar la veracidad de algunas de las afirmaciones antes expuestas, así como la existencia de semejanzas y diferencias con otros grupos de jóvenes estudiados por otros autores, en cuanto a las propuestas para solucionar o paliar los problemas actuales.

Estos jóvenes conocen muy bien la actual situación legal de los tipos de contrato existentes en el mercado laboral y, aunque desean vivamente tener para sí el indefinido, no son excesivamente críticos hacia los restantes tipos de contrato, opinando en casi un $50 \%$ que son necesarios los existentes, aunque ven pocas posibilidades de promoción y mejora en los temporales, de aprendizaje y de prácticas. Transmiten una cierta postura de conformismo con una realidad. Este cierto conformismo y aceptación de lo que hay les lleva a que, queriendo por encima de todo, tener trabajo, estén dispuestos a aceptar cualquier empleo (un $41 \%$ ) e incluso cualquier contrato o sueldo bajo ( $26 \%$ ). En cambio, no se muestran muy dispuestos a cambiar de lugar de residencia (sólo el 26\% aceptaría movilidad geográfica). Esto parece corroborar la tendencia a prolongar su situación de vinculación con la familia de origen. También se evidencia que en una buena parte de los jóvenes no hay sentimiento acuciante de necesidad de independencia, en cuanto que, o bien no están dispuestos a aceptar ningún inconveniente en el trabajo, o anteponen a éste condiciones como que les permita aprender, que sea un contrato «bueno", o que tenga elevada remuneración. Sin duda esta situación se relaciona con una «acomodada" vida familiar.

En consonancia con la opinión de que la juventud española muestra un cierto escepticismo por la política y por el asociacionismo, en este grupo resulta abrumadora la falta de interés por pertenecer a asociaciones sindicales; no sólo hay únicamente un $2.6 \%$ de afiliados a algún sindicato (cuadro 3 ), sino que casi el $45 \%$ tiene una opinión regular o mala de aquéllos y el $40 \%$ ni siquiera manifiesta una opinión. En la misma línea se muestran muy reticentes hacia los organismos oficiales, como el Instituto Nacional de Empleo, al tiempo que muy poco informados sobre otros organismos existentes (como oficinas de empleo de la Comunidad 
Autónoma) e incluso de las empresas recientemente creadas, de carácter privado. Se da una clara desconfianza hacia todo lo que tenga visos de oficialidad, redundando en el fuerte individualismo que existe. Hay una opinión de que la mejor forma de situarse es por relaciones personales 0 , en todo caso, a través de cooperativas, aunque éstas no sean apreciadas para trabajar.

CUADRO 3. AFILIACIÓN A ALGÚN SINDICATO

\begin{tabular}{|c|c|c|}
\hline SINDICATO & N. ${ }^{\circ}$ ABSOLUTO & $\begin{array}{l}\% \text { DEL TOTAL DE LA } \\
\text { ENCUESTA (542) }\end{array}$ \\
\hline Comisiones Obreras. & 6 & 1,1 \\
\hline UGT $\ldots \ldots \ldots \ldots \ldots \ldots \ldots$ & 1 & 0,2 \\
\hline 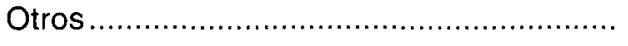 & 7 & 1,3 \\
\hline 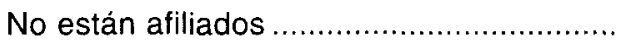 & 522 & 96,3 \\
\hline 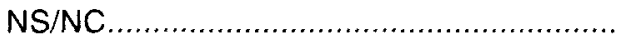 & 6 & 1,1 \\
\hline TOTAL & 542 & 100,0 \\
\hline
\end{tabular}

El individualismo se pone también de manifiesto en la idealización del trabajo por cuenta propia, que es el preferido, si pudieran optar, para un $40 \%$; en este grupo se concentraría el espíritu de empresa del conjunto. En el otro lado de la balanza está el amplio porcentaje que anhela conseguir un trabajo en empresas públicas, con sus connotaciones de seguridad, estabilidad y escasez de riesgo. Son pocos, en cambio, los que desean trabajar en empresas privadas y, en todo caso, las prefieren grandes o muy grandes. Parece existir un sentimiento generalizado de temor al empresario; bien sea por creer que en el estado actual de empleo se van a ver obligados a un exceso de dedicación, con unos resultados económicos escasos, bien por temor a que sea un trabajo precario, del que pueden ser despedidos (contratos no indefinidos) o que, incluso, puede desaparecer por cierre o quiebra de la empresa.

Ante la situación expuesta no resulta extraño que los jóvenes no parezcan muy reivindicativos, ni organizados para demandar a la sociedad cambios o puesta en marcha de determinadas acciones. Es interesante analizar los planteamientos que tienen respecto a cómo solucionar to que se considera el principal problema actual de la juventud. Para conocer su opinión se les planteó la cuestión de cómo actuar para mejorar el empleo juvenil desde el marco del Ayuntamiento, proponiéndoles algunas soluciones como investigar las necesidades para canalizar las ofertas de trabajo, 
impartir cursos relacionados con la demanda de trabajo, o cursos para nuevas profesiones, promover mejoras para dar solución a necesidades sociales y la posibilidad de exponer su libre opinión. Pues bien, pese a su escaso interés por los organismos oficiales en este punto resultó mayoritaria la opinión de que desde la Administración se estudien las necesidades y se canalicen ( $42 \%$ de respuestas), o que se den cursos para nuevas profesiones (35\%). En cambio, son muy pocos los que deciden exponer su punto de vista personal. Sólo un escaso $7 \%$ lo hace, lo que resulta ciertamente muy sintomático y muestra una postura poco reflexiva, en general. Las soluciones que en este sentido proponen no son muy imaginativas, pero sí interesantes. Pese a lo reducido de su número, se diversifican en cuatro apartados: uno se refiere a la necesidad de que se dé a los jóvenes la posibilidad de adquirir experiencia. En la enseñanza, que debería orientarse a la práctica, en la organización de cursos prácticos (con nuevas orientaciones no tradicionales) y en la creación de talleres-escuela y posibilidad de prácticas en las empresas, con percepción de algún sueldo. Incluso se reivindica la posibilidad de aprendizaje de oficios. Un $32 \%$ de las respuestas están en esta línea.

Un segundo conjunto de propuestas se refiere al deseo de que se establezcan ayudas para promoción del autoempleo, desarrollo de la iniciativa privada y de creación de empresas por jóvenes, en cooperativa, o no. En esta postura se sitúa el $22 \%$, cifra idéntica a la del tercer grupo, que propone algunas medidas drásticas para cambiar una situación que, se deduce, consideran socialmente injusta, son éstas: establecer controles sobre quienes trabajan, especialmente para evitar que haya quienes lo hagan y perciban simultáneamente el subsidio de desempleo, modificar la legislación, erradicar algún tipo de contrato, como el de aprendizaje, reducir funcionarios y bajar los sueldos más altos de los cargos públicos y promover la jubilación anticipada.

Por último, un reducido grupo propone que se creen empleos a través de una mejora de infraestructuras y de potenciar la actividad industrial o la construcción.

Aunque insistimos que son muy pocos los que arriesgan una respuesta mostrando un pequeño grado de reflexión, entre los que lo hacen parece darse un deseo generalizado de que se ayude a los jóvenes a conseguir experiencia, que se potencie el autoempleo y que se desarrollen políticas encaminadas a un reparto de trabajo y control del fraude, pero indicando muy pocas medidas concretas (sólo aparece la jubilación anticipada). 
Estas respuestas contrastan con las que en un estudio sobre los jóvenes vascos (ELZO, 1990) daban éstos a la pregunta de resolver el problema del paro, que en este caso se concentraban en la propuesta de reformas estructurales-laborales y ajustes de personal y salarios por medio de medidas como supresión de horas extraordinarias, adelanto de la edad de jubilación, reducción de la jornada laboral, aumentar el nivel tecnológico de las empresas (para hacerlas más competitivas) y las exportaciones, seguidas a notable distancia por otras como limitar a los que vienen de fuera (dando preferencia a los nacidos en la región), controlar la subida de salarios 0 , incluso (en porcentaje muy pequeño) limitar la participación de la mujer. Sólo resultan coincidentes en lo relativo al reparto del trabajo, pero en el caso de Madrid inciden mucho más en la idea de que se ayude a los jóvenes a una preparación práctica y a la búsqueda del autoempleo, que no aparece entre las del grupo vasco.

En definitiva, el sentir de este grupo de jóvenes, que es sin duda representativo de todo un conjunto social amplio, es de que existe una gran dificultad para lograr encontrar un puesto en la sociedad que les permita la independencia, lo que, además, resulta tanto más difícil cuanto menor es su preparación, sobre todo de carácter práctico. Hay un sentimiento de necesidad de ayuda en la formación o en el desarrollo de actividades de autoempleo y una sobrevaloración de los trabajos de carácter independiente, o que brinden seguridad, al tiempo que se observa un marcado individualismo, una postura de cierto conformismo y comodidad con la situación presente, que se traduce en escaso deseo de asociación y reivindicación y en una parca reflexión sobre las posibles soluciones a un problema que, por otro lado, sí ven como real y hasta acuciante.

\section{CONCLUSIÓN}

El estudio realizado a través de una encuesta a un expresivo grupo de jóvenes, nos ha permitido acercarnos al conocimiento de una realidad que no se deduce de las frías estadísticas. Varias son las conclusiones que se pueden extraer, resultando las de mayor interés, por un lado, la constatación de que los jóvenes se enfrentan a una difícil situación laboral, que se manifiesta en la escasez de oferta de empleo y sobre todo en la precariedad del poco existente, que hace que, pese a la edad tan joven de muchos, ya acumulen un buen número de empleos diversos y pasen de ocupados a parados con excesiva frecuencia. Por otro lado, se constata la 
notable dependencia familiar de muchos jóvenes que, como resultado de no disponer de facilidad para una incorporación permanente y estable a un mercado laboral, no se deciden a asumir compromisos sociales para el futuro.

Hay, desde luego, en este panorama una clara relación entre el nivel de formación y la situación laboral, de modo que sólo los más preparados consiguen una buena situación, si bien esto no significa que el estar preparado sea garantía de lograrlo, ya que también ocurre que, precisamente algunos jóvenes preparados, aunque obtengan trabajo, éste no sea el adecuado a su formación, lo que motiva que la insatisfacción y la frustración sea mayor en este grupo.

Por el contrario, pese a existir alguna relación no es muy significativa la incidencia del origen familiar en la situación laboral, en parte, porque en este caso la extracción social es bastante homogénea.

Por último, la valoración que hacen del mercado de trabajo muestra el panorama de una juventud que parece vivir con cierta comodidad, que no tiene sentimiento de fuerte necesidad de emancipación y que "acepta", un tanto pasivamente, una realidad difícil, enfrentándose al futuro con poca ilusión y escaso espíritu reivindicativo.

\section{BIBLIOGRAFIA}

- Agullera, M. J.; Azcárate, M. V.; Borderias, M. P.; Gonzalez-Yanci, M. P.; Murillo, J., y SANTOS, J. M. (1996): "Desequilibrios del mercado de trabajo juvenil en el Área Metropolitana Madrileña". Informe realizado para la CAM. Inédito.

- Ayuntamiento de MAdrid (1987): “Informe sobre el paro juvenil». Madrid.

- Casal Bataller, J. et all. (1991): “La inserción social y profesional de los jóvenes". Madrid. Ministerio de Educación y Ciencia.

- Cubeiro, J. C., y Ocaña, G. (1987): «Población, empleo y paro en la Comunidad de Madrid». Madrid. Comunidad de Madrid.

- Elzo, J. (Ed.) (1990): “Jóvenes vascos 1990. Informe sociológico sobre el comportamiento, actitudes y valores de la juventud vasca actual y de su evolución en los últimos años". Departamento de Cultura y Turismo del Gobierno Vasco. Vitoria.

- Garcia , A. (1982): “Población, empleo y paro». Madrid. Pirámide.

- Mayoral Lobato, J. (1992): "Mercado de trabajo, Políticas de empleo y Desarrollo Local». Fundación Universidad-Empresa, IRMASA. Madrid, 375 págs.

- OCDE (1993): “Perspectivas de empleo 1993". Madrid. Ministerio de Trabajo y Seguridad Social.

- OCDE/CERI (1989): "Inserción de los jóvenes en una sociedad en cambio". Madrid. Narcea, 167 págs. 